\title{
Using repetitive transcranial magnetic stimulation for the treatment of tinnitus
}

John L. Dornhoffer, MD, FACS and

Samuel D. McGill, Jr., Endowed Chair in Otolaryngology Research, University of Arkansas for Medical Sciences (UAMS), and Professor, Department of Otolaryngology-Head and Neck Surgery and Department of Neurobiology \& Developmental Sciences, UAMS

Mark Mennemeier, PhD

Director of the TMS Core Research Facility in the Center for Translational Neuroscience at UAMS and Professor, Department of Neurobiology and Developmental Sciences, UAMS

\section{INTRODUCTION}

Transcranial magnetic stimulation (TMS) has recently emerged as a potential treatment option for tinnitus. ${ }^{1-6}$ TMS induces electrical stimulation of cortical neurons by creating a brief, focused magnetic field over the surface of the brain. ${ }^{7}$ When magnetic pulses are delivered repetitively and rhythmically, the process is called repetitive TMS (rTMS).

The magnetic field induced by rTMS is brief (microseconds), relatively weak (except directly under the coil, where $1-3 \mathrm{~cm}$ of tissue is stimulated, depending on coil configuration), ${ }^{8}$ and declines rapidly with distance from the coil (falling off sharply after 2 $\mathrm{cm}) .{ }^{7,9}$ Pulse trains can be delivered at low $(\leq \mathrm{Hz})$ or high $(>1 \mathrm{~Hz})$ frequencies, which tend to decrease or increase neural activity beneath the coil, respectively. Standard TMS coils are only able to directly stimulate the superficial cortex, but deeper brain structures and structures in the opposite cerebral hemisphere may be affected by TMS via connecting neural pathways. ${ }^{10,11}$

Most studies using rTMS for the treatment of tinnitus have used low-frequency magnetic pulses applied to one temporal lobe for 30 minutes, once daily, for 3-10 consecutive days. Some have used functional imaging with neuronavigation to select the treatment site based on asymmetrical cortical activation, while others have targeted the left superior temporal lobe in all cases or the side opposite the loudest tinnitus. Regardless of targeting technique, beneficial results are obtained in about $50 \%$ of patients who receive active treatment. While treatment effects have been reported to persist for 6 months in some cases, ${ }^{2}$ most treatment studies have demonstrated tinnitus suppression effects lasting only a few days to a few weeks. ${ }^{3,5,6}$

This article will briefly review rTMS, the rationale for its use to treat tinnitus, and data relevant to its efficacy in treating tinnitus.

\section{A THEORETICAL RATIONALE}

Several theories concerning tinnitus generation have been proposed, including spontaneous otoacoustic emissions, ${ }^{12}$ thalamocortical dysrhythmia, ${ }^{13-15}$ and maladaptive cortical reorganization after peripheral injury, analogous to the model of chronic pain. ${ }^{16-18} \mathrm{~A}$ 
comprehensive working model suggests that tinnitus may be due to decreased neural inhibition at several levels of auditory processing, including the loss of normal depolarizing sensory input in auditory nuclei of the thalamus and a related process of excessive bursting activity within nuclei of the intralaminar thalamus, leading to thalamocortical dysrhythmia.

Dysrhythmia may facilitate maladaptive cortical reorganization—an increase in neural activity/representation within auditory processing areas in the temporal lobe that is often asymmetric between hemispheres-leading to phantom perception of sound $\mathrm{d}^{13,14,16,18,19}$ and creating neural contrasts, or edge effects, that enhance tinnitus perception. ${ }^{20}$ It is evident that several brain systems contribute to tinnitus phenomenology, including limbic structures, which may influence emotional aspects of tinnitus, and structures in the frontal lobe and other regions of the brain, which may influence tinnitus awareness and annoyance. Treatments for tinnitus may have effects in one or all of these brain systems.

Repetitive TMS induces electrical stimulation of cortical neurons by creating brief, focused magnetic pulses over the surface of the brain via a stimulating coil. The induced physiological effects of TMS are determined by a variety of factors, including equipment, physiologic, and anatomic variables and stimulation parameters. ${ }^{21}$ Parameters include the location, intensity, frequency, duration, and time intervals between stimulations. Stimulation parameters are constrained to some degree by safety considerations, ${ }^{22}$ but the combina tions of safe stimulation parameters that can be used are very numerous.

The effects of TMS can be excitatory or inhibitory and can be evaluated at different points in time following stimulation. ${ }^{23}$ Immediate effects are thought to result from direct excitation of inhibitory or excitatory neurons, such as a muscle twitch immediately following stimulation. Intermediate effects of TMS (occurring minutes after stimulation) may depend on changes in local pharmacology, which can have inhibitory or excitatory effects on the cortex, depending on the frequency of rTMS. Studies of the motor cortex have shown that low-frequency stimulation $(\leq \mathrm{Hz}$ ) tends to produce an inhibitory intermediate effect, ${ }^{24}$ whereas high-frequency stimulation $(>3 \mathrm{~Hz}$ ) can produce excitatory intermediate effects. ${ }^{25,26}$ The long-term effects, occurring over days to months, generally concern treatment studies. Relatively less is known about the neurobiologic mechanisms involved in this class of studies. This is unfortunate, as these types of changes are most relevant to studies that use rTMS to treat tinnitus.

Low-frequency rTMS applied over the temporal cortex is most commonly used to treat tinnitus. In a typical application, the stimulating coil is placed over one temporal lobe and magnetic pulses are delivered once per second for 30 minutes, once per day for 3-10 consecutive days. The rationale for this approach appears to be rooted in theories that view tinnitus as stemming from increased neural activity within the temporal lobe. Applying lowfrequency rTMS over these areas would be expected to reduce tinnitus by inhibiting activity beneath the stimulating coil.

This rationale also underlies the use of PET and other imaging techniques to navigate the stimulating coil over areas within the temporal cortex that show increased metabolic activity. This rationale, however, does not stand up against certain empirical observations, which are reviewed below.

Other approaches using rTMS to treat tinnitus have used combinations of high- and lowfrequency stimulation delivered to the frontal and temporal cortex, respectively. ${ }^{27}$ Different types of burst frequencies have also been applied in patients with tinnitus. The rationale for this approach is that burst stimulation may activate neurons more powerfully than tonic stimulation, ${ }^{28}$ and bursts may activate neurons that are not activated by tonic stimulation. ${ }^{29}$ 
It is possible, therefore, that rTMS treatment may work by "normalizing" or "synchronizing" the oscillatory activity that results in edge effects and cortical reorganization or by altering the balance of activity in brain systems that are interconnected with auditory processing areas, or both. Repeated applications of rTMS may produce longer lasting modulation of cortical activity and interconnected brain systems.

\section{EFFICACY OF rTMS FOR THE TREATMENT OF TINNITUS}

\section{Review of studies}

Studies using rTMS to investigate and treat tinnitus (see Langguth et al. for a review ${ }^{30}$ ) can be divided into: (1) those involving single sessions to disrupt tinnitus transiently for the purpose of investigating TMS parameters and locations for stimulation ${ }^{29,31-37}$ and (2) those using repeated sessions of rTMS on consecutive days in an effort to induce a lasting modulation of tinnitus-related neural activity as a potential therapeutic application. ${ }^{2,3,5,6,38-43}$

Single-session studies typically used trains of high-frequency rTMS $(10-20 \mathrm{~Hz})$ to produce an immediate, shortlasting interruption of tinnitus perception. More recently, the short-term effects of single sessions of theta, alpha, and beta burst rTMS on tinnitus were investigated in patients with tinnitus. ${ }^{37,44}$ Burst rTMS appeared to suppress narrow band/ white noise tinnitus better than did tonic stimulation, but no difference between the two types of stimulation was observed in pure-tone tinnitus.

In contrast to single-session studies, those using repeated sessions of rTMS to treat tinnitus have almost exclusively relied on low-frequency (1 Hz) rTMS. ${ }^{30,45}$ Most of these studies used long trains of magnetic pulses (1200-2000) repeated over 5 to 10 consecutive days. Beneficial results were obtained in about $50 \%$ of patients who receive active treatment (sham stimulation had a much lower response rate). The 50\% response rate appeared to hold whether stimulation was applied to the left or right hemisphere.

In the only treatment study comparing frequency of stimulation, Khedr et al. used low- and high-frequency stimulation over the temporal cortex to treat tinnitus in 66 patients (i.e., 1500 pulses over the temporoparietal cortex at 1,10 , and $25 \mathrm{~Hz}$ and $100 \%$ of MT $\times 10$ days versus sham stimulation). ${ }^{40,46}$ Patients were followed for 4 months and 1 year. Each stimulation frequency, except for sham, was associated with lasting improvement on the tinnitus handicap questionnaire, but not the tinnitus masking procedure.

Several studies used PET scans to target 1-Hz rTMS over areas of excessive asymmetric cortical activity in the left or right temporal cortex..$^{2,3,6,33,47}$ Despite some very promising initial claims of long-term efficacy, ${ }^{2}$ subsequent investigations observed short-term effects ( $<2$ weeks) even when rTMS treatment was applied for 10 rather than 5 days. ${ }^{3}$ Additionally, a recent review of coil navigation methods in studies of tinnitus indicated that simply placing the coil over a standard location in the left temporal cortex was as efficacious as guiding placement of the coil with PET or other techniques. ${ }^{38,45}$

The empirical data from Langguth's review of coil navigation techniques ${ }^{45}$ and Khedr et al. ${ }^{40,46}$ cast doubt on the simple hypothesis that tinnitus is promoted by increased, asymmetric neural activity within the temporal cortex, especially as high-frequency rTMS would be expected to excite rather than inhibit neural activity beneath the stimulation coil.

\section{Study of maintenance rTMS}

Because a single course of rTMS tends to reduce tinnitus perception for only a short period of time,, 46 we have examined the application of "maintenance rTMS." 48 Our preliminary 
data showed that maintenance rTMS could improve the duration of the treatment effect. Currently, we have five patients who were selected for maintenance treatment because they demonstrated an initial positive response to a treatment course of 1-Hz rTMS.

All these patients showed a replication of the initial treatment response following a threesession course of maintenance rTMS (1800 pulses delivered at $1 \mathrm{~Hz}$ and $110 \%$ of MT for 3 consecutive days). Patients were then asked to contact us for additional courses of maintenance treatment when and if their tinnitus returned.

One patient reported a subjective return of tinnitus and received four courses of maintenance treatment over the right temporal lobe. In this patient, tinnitus remained absent in the left ear and near baseline in the right ear 6 months after the last maintenance treatment ended. Moreover, the patient reports that tinnitus is largely unnoticeable in daily life.

Two patients did not call to request additional treatment after their second maintenance session and indicated in follow-up e-mails that their tinnitus remained lower and was less annoying.

The remaining two patients are still being followed. One has now received three courses of maintenance treatment extending over one year. Tinnitus perception in this patient is rated as lower and less annoying in between the courses of maintenance treatment than at pretreatment assessment.

These pilot data demonstrate the feasibility of using maintenance treatment to treat tinnitus chronically and show that three rTMS sessions are sufficient to replicate the initial treatment effect, with benefits extending from weeks to months.

\section{Measuring change in tinnitus before and after rTMS}

The efficacy of rTMS for tinnitus may depend on how tinnitus is assessed. Early reports on the efficacy of rTMS for tinnitus relied on questionnaires to evaluate change, reporting high treatment response rates (up to 80\%) and long treatment effects (up to 6 months after one course of treatment). ${ }^{2}$

Subsequent studies that incorporated visual analogue ratings of tinnitus loudness observed response rates around 50\% and shorter treatment duration ( $<2$ months). ${ }^{3,5,6}$ For example, the Khedr et al. study ${ }^{46}$ reported long-term change for questionnaire data, which assess the impact of tinnitus on a patient's life, but not for assessments based on a tinnitus masking procedure, which is a more direct assessment of tinnitus loudness. These disassociations in assessing tinnitus and response to treatment can be addressed by using a combination of questionnaire, visual analogue, and tinnitus loudness matching procedures.

Finally, many patients are in the habit of ignoring tinnitus, and they may not be well prepared to assess change when it occurs. Therefore, patients in any treatment trial using rTMS should be required to establish a stable baseline of tinnitus ratings and loudness matching prior to any intervention.

\section{CONCLUSION}

Although very promising results have been obtained to date, in order for rTMS to serve as a tinnitus therapy, certain parameters, such as frequency, duration, and number of treatment sessions; targeting strategies; delivery; and patient factors need to be better understood. These are currently under investigation in several clinical trials. 


\section{Acknowledgments}

Our work is supported by the National Center for Research Resources (RR20146 \& 1UL1RR029884); National Institute of Child Health and Human Development (HD040631 \& HD055269); and by a Tinnitus Research Consortium grant-in-aid.

\section{REFERENCES}

1. Dornhoffer JL, Mennemeier M. Transcranial magnetic stimulation and tinnitus: Implications for theory and practice. J Neurol Neurosurg Psychiatr. 2007; 78:113. [PubMed: 17229742]

2. Kleinjung T, Eichhammer P, Langguth B, et al. Long-term effects of repetitive transcranial magnetic stimulation (rTMS) in patients with chronic tinnitus. Otolaryngol Head Neck Surg. 2005; 132:566-569. [PubMed: 15806046]

3. Plewnia C, Reimold M, Najib A, et al. Moderate therapeutic efficacy of positron emission tomography-navigated repetitive transcranial magnetic stimulation for chronic tinnitus: A randomised, controlled pilot study. J Neurol Neurosurg Psychiatr. 2007; 78:152-156. [PubMed: 16891384]

4. Richter GT, Mennemeier M, Bartel T, et al. Repetitive transcranial magnetic stimulation for tinnitus: A case study. Laryngoscope. 2006; 116:1867-1872. [PubMed: 17016213]

5. Rossi S, De Capua A, Ulivelli M, et al. Effects of repetitive transcranial magnetic stimulation on chronic tinnitus: A randomised, crossover, double blind, placebo controlled study. J Neurol Neurosurg Psychiatr. 2007; 78:857-863. [PubMed: 17314192]

6. Smith JA, Mennemeier M, Bartel T, et al. Repetitive transcranial magnetic stimulation for tinnitus: A pilot study. Laryngoscope. 2007; 117:529-534. [PubMed: 17334317]

7. Bohning, DE. Introduction and Overview of TMS Physics. Washington, DC: American Psychiatric Press; 2000. p. 13-44.

8. Cohen LG, Roth BJ, Nilsson J, et al. Effects of coil design on delivery of focal magnetic stimulation. Technical considerations. Electroencephalogr Clin Neurophysiol. 1990; 75:350-357. [PubMed: 1691084]

9. Roth BJ, Momen S, Turner R. Algorithm for the design of magnetic stimulation coils. Med Biol Eng Comput. 1994; 32:214-216. [PubMed: 8022220]

10. Kimbrell TA, Little JT, Dunn RT, et al. Frequency dependence of antidepressant response to left prefrontal repetitive transcranial magnetic stimulation (rTMS) as a function of baseline cerebral glucose metabolism. Biol Psychiatr. 1999; 46:1603-1613.

11. Fox P, Ingham R, George MS, et al. Imaging human intra-cerebral connectivity by PET during TMS. Neuroreport. 1997; 8:2787-2791. [PubMed: 9295118]

12. Bernal B, Altman NR. Auditory functional MR imaging. Am J Roentgenol. 2001; 176:1009-1015. [PubMed: 11264100]

13. Jeanmonod D, Magnin M, Morel A. Low-threshold calcium spike bursts in the human thalamus. Common physiopathology for sensory, motor and limbic positive symptoms. Brain. 1996; 119(Pt 2):363-375. [PubMed: 8800933]

14. Llinas R, Ribary U, Jeanmonod D, et al. Thalamocortical dysrhythmia I. Functional and imaging aspects. Thal \& Related Syst. 2001; 1:237-244.

15. Llinas RR, Ribary U, Jeanmonod D, et al. Thalamocortical dysrhythmia: A neurological and neuropsychiatric syndrome characterized by magnetoencephalography. Proc Nat Acad Sci USA. 1999; 96:15222-15227. [PubMed: 10611366]

16. Muhlnickel W, Elbert T, Taub E, Flor H. Reorganization of auditory cortex in tinnitus. Proc Nat Acad Sci USA. 1998; 95:10,340-10,343.

17. Rauschecker JP. Auditory cortical plasticity: A comparison with other sensory systems. Trends Neurosci. 1999; 22:74-80. [PubMed: 10092047]

18. Moller AR. Similarities between chronic pain and tinnitus. Am J Otol. 1997; 18:577-585. [PubMed: 9303153]

19. Kadner A, Viirre E, Wester DC, et al. Lateral inhibition in the auditory cortex: An EEG index of tinnitus? Neuroreport. 2002; 13:443-446. [PubMed: 11930157] 
20. Llinas R, Urbano FJ, Leznik E, et al. Rhythmic and dysrhythmic thalamocortical dynamics: GABA systems and the edge effect. Trends Neurosci. 2005; 28:325-333. [PubMed: 15927689]

21. Sack AT, Linden DE. Combining transcranial magnetic stimulation and functional imaging in cognitive brain research: Possibilities and limitations. Brain Res Brain Res Rev. 2003; 43:41-56. [PubMed: 14499461]

22. Wassermann EM. Risk and safety of repetitive transcranial magnetic stimulation: Report and suggested guidelines from the International Workshop on the Safety of Repetitive Transcranial Magnetic Stimulation, June 5-7, 1996. Electroencephalogr Clin Neurophysiol. 1998; 108:1-16. [PubMed: 9474057]

23. George MS, Nahas Z, Kozel FA, et al. Mechanisms and state of the art of transcranial magnetic stimulation. J Ect. 2002; 18:170-181. [PubMed: 12468991]

24. Chen R, Classen J, Gerloff C, et al. Depression of motor cortex excitability by low-frequency transcranial magnetic stimulation. Neurology. 1997; 48:1398-1403. [PubMed: 9153480]

25. Pascual-Leone A, Tormos JM, Keenan J, et al. Study and modulation of human cortical excitability with transcranial magnetic stimulation. J Clin Neurophysiol. 1998; 15:333-343. [PubMed: 9736467]

26. Wu T, Sommer M, Tergau F, Paulus W. Lasting influence of repetitive transcranial magnetic stimulation on intracortical excitability in human subjects. Neurosci Lett. 2000; 287:37-40. [PubMed: 10841985]

27. Londero A, Langguth B, De Ridder D, et al. Repetitive transcranial magnetic stimulation (rTMS): A new therapeutic approach in subjective tinnitus? Neurophysiol Clin. 2006; 36:145-155. [PubMed: 17046609]

28. Huang YZ, Edwards MJ, Rounis E, et al. Theta burst stimulation of the human motor cortex. Neuron. 2005; 45:201-206. [PubMed: 15664172]

29. De Ridder D, van der Loo E, Van der Kelen K, et al. Do tonic and burst TMS modulate the lemniscal and extralemniscal system differentially? Int J Med Sci. 2007; 4:242-246. [PubMed: 17952200]

30. Langguth B, de Ridder D, Dornhoffer JL, et al. Controversy: Does repetitive transcranial magnetic stimulation/transcranial direct current stimulation show efficacy in treating tinnitus patients? Brain Stim. 2008; 1:192.

31. Plewnia C, Reimold M, Najib A, et al. Dose-dependent attenuation of auditory phantom perception (tinnitus) by PET-guided repetitive transcranial magnetic stimulation. Hum Brain Mapp. 2007; 28:238-246. [PubMed: 16773635]

32. Londero A, Lefaucheur JP, Malinvaud D, et al. Magnetic stimulation of the auditory cortex for disabling tinnitus: Preliminary results. Presse Med. 2006; 35:200-206. [PubMed: 16493347]

33. De Ridder D, Verstraeten E, Van der Kelen K, et al. Transcranial magnetic stimulation for tinnitus: Influence of tinnitus duration on stimulation parameter choice and maximal tinnitus suppression. Otol Neurotol. 2005; 26:616-619. [PubMed: 16015156]

34. Plewnia C, Bartels M, Gerloff C. Transient suppression of tinnitus by transcranial magnetic stimulation. Ann Neurol. 2003; 53:263-266. [PubMed: 12557296]

35. Fregni F, Marcondes R, Boggio PS, et al. Transient tinnitus suppression induced by repetitive transcranial magnetic stimulation and transcranial direct current stimulation. Eur J Neurol. 2006; 13:996-1001. [PubMed: 16930367]

36. Folmer RL, Carroll JR, Rahim A, et al. Effects of repetitive transcranial magnetic stimulation (rTMS) on chronic tinnitus. Acta Otolaryngol Suppl. 2006:96-101. [PubMed: 17114152]

37. De Ridder D, van der Loo E, Van der Kelen K, et al. Theta, alpha and beta burst transcranial magnetic stimulation: Brain modulation in tinnitus. Int J Med Sci. 2007; 4:237-241. [PubMed: 17952199]

38. Langguth B, Zowe M, Landgrebe M, et al. Transcranial magnetic stimulation for the treatment of tinnitus: A new coil positioning method and first results. Brain Topogr. 2006; 18:241-247. [PubMed: 16845596]

39. Kleinjung T, Steffens T, Sand P, et al. Which tinnitus patients benefit from transcranial magnetic stimulation? Otolaryngol Head Neck Surg. 2007; 137:589-595. [PubMed: 17903575] 
40. Khedr EM, Rothwell JC, Ahmed MA, El-Atar A. Effect of daily repetitive transcranial magnetic stimulation for treatment of tinnitus: Comparison of different stimulus frequencies. J Neurol Neurosurg Psychiatr. 2008; 79:212-215. [PubMed: 18202212]

41. Langguth B, Kleinjung T, Frank E, et al. High-frequency priming stimulation does not enhance the effect of low-frequency rTMS in the treatment of tinnitus. Exp Brain Res. 2008; 184:587-591. [PubMed: 18066684]

42. Lee SL, Abraham M, Cacace AT, Silver SM. Repetitive transcranial magnetic stimulation in veterans with debilitating tinnitus: A pilot study. Otolaryngol Head Neck Surg. 2008; 138:398399. [PubMed: 18312892]

43. Kleinjung T, Eichhammer P, Landgrebe M, et al. Combined temporal and prefrontal transcranial magnetic stimulation for tinnitus treatment: A pilot study. Otolaryngol Head Neck Surg. 2008; 138:497-501. [PubMed: 18359361]

44. Poreisz C, Paulus W, Moser T, Lang N. Does a single session of theta-burst transcranial magnetic stimulation of inferior temporal cortex affect tinnitus perception? BMC Neurosci. 2009; 10:54. [PubMed: 19480651]

45. Langguth B, Kleinjung T, Landgrebe M, et al. rTMS for the treatment of tinnitus: The role of neuronavigation for coil positioning. Clin Neurophysiol. 2010; 40:45-48.

46. Khedr EM, Rothwell JC, El-Atar A. One-year follow up of patients with chronic tinnitus treated with left temporoparietal rTMS. Eur J Neurol. 2009; 16:404-408. [PubMed: 19175380]

47. Langguth B, Eichhammer P, Wiegand R, et al. Neuronavigated rTMS in a patient with chronic tinnitus. Effects of 4 weeks treatment. Neuroreport. 2003; 14:977-980. [PubMed: 12802186]

48. Mennemeier M, Chelette KC, Myhill J, et al. Maintenance repetitive transcranial magnetic stimulation can inhibit the return of tinnitus. Laryngoscope. 2008; 118:1228-1232. [PubMed: $18475211]$ 